\title{
PARAMETRIC BEAM INSTABILITY OF RELATIVISTIC CHARGED PARTICLES IN A CRYSTAL
}

\author{
V.G. BARYSHEVSKY and I.D. FERANCHUK \\ Department of Physics, Byelorussian State University, Minsk-80, USSR
}

Received 14 September 1983

Revised manuscript received 27 February 1984

\begin{abstract}
A new mechanism of the instability of an electron (positron) beam under conditions of the parametric X-rays is discovered. Equations, which define the amplitude increment of the instability and the starting current, are obtained. It is shown that a considerable part of the particle energy transforms into coherent X-ray energy due to this parametric instability.
\end{abstract}

Known schemes of coherent $\mathrm{X}$-ray and gamma-ray sources are basically connected with the creation of the conditions, when resonant transitions of Mössbauer nuclei or the internal electron shell of heavy atoms have an inverse population. It was shown in refs. $[1,2]$ that these conditions were realized for a relatively small threshold current $\left(j \gtrless 10^{2} \mathrm{~A} / \mathrm{cm}^{2}\right)$, when parametric $X$-rays $[2,3]$ from the modulated electron beam were used. But in this scheme a solu. tion of the very complicated problem of X-ray modulation of the ultrarelativistic particle beam is required.

An essentially different mechanism of the coherent X-ray generation is considered in the present paper. It is shown that parametric X-rays lead to longitudinal self-modulation of the relativistic channelled particle beam and as a result the parametric beam instability arises. In this process the longitudinal energy of the particles efficiently transforms into the energy of the coherent monochromatic X-rays. The mechanism considered is in many aspects analogous to the processes which are used in the powerful generators of superhigh-frequency radiation and freeelectron lasers [4-7].

The interaction of the channelled particle beam with a radiation field and the crystal is described by the following self-consistent system of the Maxwell equations and the particle's equations of motion

$$
\begin{aligned}
& k^{2} \mathcal{E}_{\alpha}(k, \omega)-k_{\alpha}(k \cdot \mathcal{E}) \\
& \quad-\omega^{2} \sum_{\tau} \epsilon_{\alpha \beta}\left(k, k_{\tau}, \omega\right) \varepsilon_{\beta}\left(k_{\tau}, \omega\right) \\
& \quad=4 \pi \mathrm{i} \omega j_{\alpha}(k, \omega), \\
& m \mathrm{~d} \mathbf{v}_{i} / \mathrm{d} t-\gamma^{-1} \boldsymbol{F}\left(r_{i}\right) \\
& \quad=-(e / \gamma)\left[\mathcal{E}\left(r_{i}, t\right)+v_{i} \times B\left(r_{i}, t\right)-v_{i}\left(v_{i} \cdot \mathcal{E}\right)\right], \\
& \hbar=c=1, \quad e^{2}=1 / 137, \quad k_{\tau}=k+\tau, \quad \gamma=E / m,
\end{aligned}
$$

where $r_{i}$ and $v_{i}$ are the coordinate and velocity of the $i$ th particle of the beam; $E(t)$ is the total energy of the particle at the time $t ; \epsilon_{\alpha \beta}\left(k, k_{\tau} ; \omega\right)$ are the Fourier components of the dielectric constant of the crystal [2]; $\boldsymbol{t}$ is the reciprocal lattice vector of the crystal, $\mathcal{E}(r, t)$ is the electric radiation field:

$$
\varepsilon(\boldsymbol{r}, t)=\int \mathrm{d} \boldsymbol{k} \mathrm{d} \omega \boldsymbol{\varepsilon}(\boldsymbol{k}, \omega) \exp (\mathrm{i} \boldsymbol{k} \cdot \boldsymbol{r}-\mathrm{i} \omega t),
$$

and $j(r, t)$ is the current density which may be written as

$j(\boldsymbol{r}, t)=e \sum_{i} \mathbf{v}_{i} \delta\left[\boldsymbol{r}-\boldsymbol{r}_{i}(t)\right]$.

Let us consider the simplest case of planar channelling, when the interplane potential is periodic along the $X$-axis with period $d$ (see fig. 1). Then the 


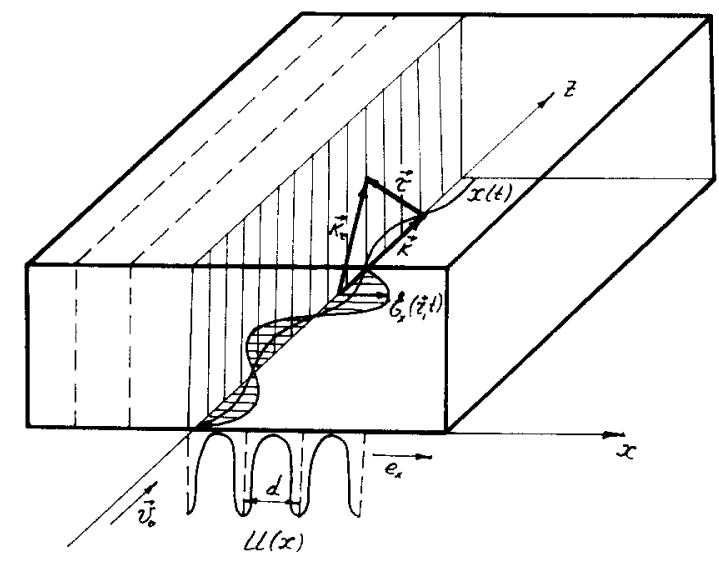

Fig.1. Arrangement of the crystal, particle beam and radiation field.

equation of motion of the particle in a channel has the form

$r_{i}(t)=v_{0} t+e_{x} x_{i}(t)+r_{i 0}$,

$F\left(r_{i}\right)=-e_{x} \partial U / \partial x_{i}, \quad e_{x}^{2}=1$,

where $r_{i 0}$ and $v_{0}$ are the initial coordinate and velocity of the $i$ th particle and $v_{0}$ is directed along the $Z$ axis.

In order to investigate the considered instability of the system in accordance with the general theory (see, for example, refs. [4-7]), one has to solve eqs. (1), (2) in a linear approximation under the radiation field $\mathcal{E}(r, t)$ and to find the dispersion equation which defines the propagation of the eigenwaves in the crystal with the channelled electron (positron) beam. In the zeroth-order approximation the motion of the channelled particle $x_{0}(t)$ is periodic with frequency $\omega_{0}$ depending on the transversal particle energy $\epsilon=\frac{1}{2} E v_{x}^{2}+U\left(x_{0}\right)$. The oscillation phase is arbitrary and therefore the average current connected with the function $x_{0}(t)$ is equal to zero. With this motion only spontaneous channelling radiation occurs, which is incoherent, with intensity being proportional to the first order of the particle current [8].

But if one takes into account the first-order correction $x^{(1)}(t)$, connected with the interaction of the particle with the radiation field of frequency $\omega$, longitudinal modulation of the beam arises. As a consequence the Fourier component of the current $j^{(1)}(\omega)$ is nonzero and, in its turn, leads to coherent radiation with intensity proportional to $\left[j^{(1)}\right]^{2}$. The beam in- stability is caused by such an interaction between particles and radiation field.

Let us consider this process quantitatively. The first-order correction $x^{(1)}(t)$ is defined by the following expression averaged over the initial phase of the oscillation

$$
\begin{aligned}
& \overline{x^{(1)}(t)}=\mathrm{i} \omega_{0} \int \mathrm{d} \boldsymbol{k} \mathrm{d} \omega \exp \left(\mathrm{i} \boldsymbol{k} \cdot \boldsymbol{r}_{0}\right) \exp \left(-\mathrm{i} \Omega_{k} t\right) \\
& \quad \times\left\{\left[\Omega_{k} / \omega-\overline{v_{x}^{2}}\right] \varepsilon_{x}(\boldsymbol{k}, \omega)+v_{0}\left(k_{x} / \omega\right) \varepsilon_{z}(\boldsymbol{k}, \omega)\right\} \\
& \quad \times \frac{\partial}{\partial \epsilon}\left(\sum_{n} n\left|x_{n}\right|^{2} \frac{1}{\mathrm{i}\left(\Omega_{k}-n \omega_{0}\right)-\nu}\right) \\
& \Omega_{k}=\omega-k_{z} v_{0}, \quad \overline{v_{x}^{2}}=\sum_{n} \omega_{0}^{2} n^{2}\left|x_{n}\right|^{2} .
\end{aligned}
$$

Using formula (5) in eq. (3) one can calculate the current and charged density of the beam

$$
\begin{aligned}
& j_{x}^{(1)}=n_{0} e^{2} \omega_{0}\left[\left(\Omega_{k} / \omega-\overline{v_{x}^{2}}\right) \varepsilon_{x}+v_{0}\left(k_{x} / \omega\right) \varepsilon_{z}\right] \\
& \times \frac{\partial}{\partial \epsilon}\left(\sum_{n} \omega_{0} \frac{n^{2}\left|x_{n}\right|^{2}}{\mathrm{i}\left(\Omega_{k}-n \omega_{0}\right)-\nu}\right), \\
& j_{z}^{(1)}=n_{0} e^{2} \omega_{0}\left\{\left[\Omega_{k} / \omega-\overline{v_{x}^{2}}\right] \varepsilon_{x}+v_{0}\left(k_{x} / \omega\right) \varepsilon_{z}\right\} \\
& \quad \times \frac{\partial}{\partial \epsilon}\left(\sum_{n} \frac{k_{x} v_{0} n\left|x_{n}\right|^{2}}{\mathrm{i}\left(\Omega_{k}-n \omega_{0}\right)-\nu}\right) ; \quad \rho^{(1)}=v_{0}^{-1} j_{z}^{(1)},
\end{aligned}
$$

and the Maxwell equation (1) transforms as follows:

$$
\begin{aligned}
& k^{2} \varepsilon_{\alpha}(\boldsymbol{k}, \omega)-k_{\alpha}(\boldsymbol{k} \cdot \boldsymbol{\varepsilon}) \\
& -\omega^{2} \sum_{\tau} \epsilon_{\alpha \beta}\left(\boldsymbol{k}, \boldsymbol{k}_{\tau}, \omega\right) \varepsilon_{\beta}\left(\boldsymbol{k}_{\tau}, \omega\right) \\
& -\omega^{2} \sigma_{\alpha \beta}(\boldsymbol{k}, \omega) \varepsilon_{\beta}(\boldsymbol{k}, \omega)=0
\end{aligned}
$$

where the quantities $\delta_{\alpha \beta}+\sigma_{\alpha \beta}(k, \omega)$ define the dielectric constant of the beam,

$$
\begin{aligned}
& \sigma_{x x}=\lambda\left(\Omega_{k} / \omega-v_{x}^{2}\right) \Delta, \quad \sigma_{x z}=\lambda v_{0}\left(k_{x} / \omega\right) \Delta, \\
& \sigma_{z z}=\lambda v_{0}\left(k_{x}^{2} / \omega\right)\left(\Omega_{k} v_{0}-k_{z} / \gamma^{2}\right) \Delta_{1}, \\
& \left.\sigma_{z x}=\lambda k_{x}\left[\Omega_{k} v_{0}-k_{z} / \gamma^{2}\right)\right]\left(\Omega_{k} / \omega-\overline{v_{x}^{2}}\right) \Delta_{1}, \\
& \lambda=4 \pi e^{2} n_{0} \omega_{0} / m \gamma \omega^{2}=\left(\omega_{\mathrm{p}}^{2} / \omega^{2}\right) \omega_{0},
\end{aligned}
$$


$\Delta=E \frac{\partial}{\partial \epsilon}\left(\sum_{n} n\left|x_{n}\right|^{2} \frac{\omega \omega_{0} n-k_{x}^{2}}{\Omega_{k}-n \omega_{0}+\mathrm{i} \nu}\right)$,

$\Delta_{1}=E \frac{\partial}{\partial \epsilon}\left(\sum_{n} \frac{n\left|x_{n}\right|^{2}}{\Omega_{k}-n \omega_{0}+\mathrm{i} \nu}\right)$,

$\gamma^{-2}=1-v_{0}^{2}, \quad v=L^{-1}$

where $L$ is the length of the crystal; $n_{0}$ is the particle density of the incident beam; $x_{n}$ is the Fourier component of the function $x_{0}(t)$ corresponding to the frequency $n \omega_{0}$, from the quantum point of view this value coincides with the matrix element of the coordinate operator between the energy bands of the channelled particle [9]; $\bar{v}_{x}^{2}$ is the square of the particle transversal velocity averaged on the oscillation phase $; \omega_{p}$ is the plasma frequency of the beam. The electron beam is an anisotropic medium, because the transversal and longitudinal motions of the electrons are not equivalent. As a result the dielectric constant tensor is nondiagonal and the electromagnetic field is nontransversal in the medium. But we shall see later that the components $\sigma_{x z}, \sigma_{z x}$ and $\sigma_{z z}$ may be neglected for real values of the beam current.

Let us use now the two-beam approximation of the dynamical theory of X-ray diffraction for the solution of eq. (7). Choose the geometry of the problem in such a way that the oscillation direction ( $X$ axis) is perpendicular to the diffraction plane, formed by the vectors $\boldsymbol{k}$ and $\boldsymbol{\tau}$ (fig. 1). Then the above-mentioned dispersion equation has the form

$$
\begin{aligned}
& {\left[k^{2}-\omega^{2}\left(1+g_{0}\right)\right]\left[k_{\tau}^{2}-\omega^{2}\left(1+g_{1}\right)\right]-\omega^{4} g_{\tau} g_{-\tau}} \\
& =\omega^{2}\left[k_{\tau}^{2}-\omega^{2}\left(1+g_{1}\right)\right] \\
& \quad \times\left(\sigma_{x x}+\frac{\omega^{2} \sigma_{x z} \sigma_{z x}}{k^{2}-\omega^{2}\left(1+g_{0}\right)-\omega^{2} \sigma_{z z}}\right),
\end{aligned}
$$

where $g_{0}, g_{1}, g_{\tau}$ are defined by the crystal dielectric constant tensor $\epsilon_{\alpha \beta}$ and are directly connected with the amplitude of the photon coherent scattering on the crystal atoms and nuclei [2].

One can easily estimate that the refraction of the emitted photons by the crystal is more essential than the latter by the particle beam when $j \lesssim 10^{8} \mathrm{~A} / \mathrm{cm}^{2}$. It means that $\left|g_{\lambda}\right| \gg\left\|\sigma_{\alpha \beta}\right\|$ and the term $\sim \sigma_{\alpha \beta}^{2}$ in eq. (9) can be neglected. As a result the instability de- pends on the component $\sigma_{x x}$ only.

Let us first consider the condition of instability in the case when the radiation frequency does not satisfy the parametric radiation condition $[2,3]$ and the photon diffraction is not essential. In this case eq. (9) has the form

$$
\begin{aligned}
k^{2} & -\omega^{2}\left(1+g_{0}\right) \\
& =\omega_{0} \omega_{\mathrm{p}}\left[\Omega_{k} / \omega-v_{x}^{2}\right] \frac{\left|x_{1}\right|^{2}\left(\omega \omega_{0}-k_{x}^{2}\right)}{\left(\omega-k_{z} v_{0}-\omega_{0}+\mathrm{i} \nu\right)^{2}} \frac{\partial \omega_{0}}{\partial \epsilon},
\end{aligned}
$$

which is typical of the theory of plasma superhighfrequency generators [5] and the free-electron laser [7]. But the radiation spectrum of the system considered is continuous because of the absence of a resonator. According to the general theory of such equations [5-7] the beam instability with respect to electromagnetic radiation takes place near the resonant values $\omega_{\mathrm{r}}$ and $k_{z \mathrm{r}}$ which are solutions of the following equations

$k^{2}-\omega^{2}\left(1+g_{0}\right)=0$,
$\omega-k_{z} v_{0}-\omega_{0}=0$.

The amplitude increment of the instability is defined by the imaginary part $\delta=\operatorname{Im} k_{z}(\omega)$ of the solution of eq. (10) and is

$$
\begin{aligned}
& \delta=-\frac{1}{2} \sqrt{3} Q^{1 / 3}, \\
& Q=\left.\left|\frac{E \omega_{\mathrm{p}}^{2} \omega_{0}}{2 \omega_{\mathrm{r}}}\left(\Omega_{k} / \omega_{\mathrm{r}}-\bar{v}_{x}^{2}\right)\right| x_{1}\right|^{2}\left(\omega_{0} \omega_{\mathrm{r}}-k_{x}^{2}\right) \frac{\partial \omega_{0}}{\partial \epsilon} \mid .
\end{aligned}
$$

The starting current $j_{\mathrm{s}}$ (the self-excitation condition) is defined by the following inequality

$$
|\delta| L_{\mathrm{abs}}>1, \quad|\delta|\left[2 \omega_{\mathrm{r}} \operatorname{Im} g_{0}\left(\omega_{\mathrm{r}}\right)\right]^{-1}>1,
$$

where $L_{\mathrm{abs}}$ is the absorption length for the radiation with frequency $\omega_{\mathrm{r}}$. One can find from eqs. (13),(14) the following estimation for the current

$j_{\mathrm{s}} \gtrsim 10^{12} \mathrm{~A} / \mathrm{cm}^{2}$, when $\omega_{\mathrm{r}}=10 \mathrm{keV}, \gamma=100$.

This estimation shows that coherent X-ray generation from the channelled particles is not realized in practice in the case considered. But an essentially differ- 
ent result is obtained when the parametric X-ray condition is satisfied $[2,3]$. In this case the emitted photons are diffracted by the crystal and the resonant values $\omega_{\mathrm{r}}$ and $k_{z \mathrm{r}}$ satisfy both eqs. (11), (12) and the Bragg condition

$(\boldsymbol{k}+\boldsymbol{\tau})^{2}=k^{2}$.

As a consequence $\omega_{\mathrm{r}}$ and $\boldsymbol{k}_{\mathrm{r}}$ are unequivocally defined by eqs. (11), (12), (15) and the amplitude increment of the parametric instability and the self-excitation condition are obtained from the general equation (9) in the form

$\delta=-2^{-1 / 2}\left(Q \omega_{\mathrm{r}}\left|g_{0}\right|\right)^{1 / 4}$,

$\frac{1}{2}|\delta| \omega_{\mathrm{r}}^{-1}\left[\operatorname{Im}\left(g_{0}-\sqrt{g_{\tau} g_{-\tau}}\right)\right]^{-1}>1$.

One can find the following estimation from eq. (16) under the above-mentioned conditions:

$j_{\mathrm{s}} \gtrsim 10^{7} \mathrm{~A} / \mathrm{cm}^{2}$.

Such a value of the current density is realized in the current bunches of modern accelerators.

The physical reasons for the decrease of the starting current are:

(a) if the parametric X-ray condition (15) is fulfilled, the crystal is actually a resonator for the emitted photons and this fact permits one to realize the one-mode generation regime;

(b) the spectral density of the radiation for the parametric $X$-rays is essentially higher than the latter for the channelling radiation [2];

(c) the radiation absorption length is increased in the parametric X-ray condition because of the Borrmann effect [2].
The greatest transformation of the longitudinal energy of the particle into the radiation energy under the instability condition is caused by capture of the particles in the potential pits of the progressive radiation waves [5]. It is possible when the particle velocity $v_{0}$ is greater than the $\mathrm{X}$-ray phase velocity in the crystal. According to ref. [2] this condition is fulfilled when the particle energy is

$E>E_{0}=m\left|g_{0}\right|^{-1 / 2}$.

Then the greatest energy of the coherent X-rays emitted by a particle is $E_{\mathrm{rad}}=E-E_{0}$. A detailed calculation of the considered generator efficiency demands the investigation of the non-linear solution of the general equations (1), (2).

\section{References}

[1] V.G. Baryshevsky and I.D. Feranchuk, Abstr. XIth Cont. on Charged particle collisions in crystals, Moscow State University, (1982) p. 213.

[2] V.G. Baryshevsky and 1.D. Feranchuk, J. Phys. (Paris) 44 (1983) 913.

[3] V.G. Baryshevsky and I.D. Feranchuk, Phys. Lett. 57 A (1976) 183

[4] A.V. Gaponov, M.I. Petelin and V.K. Yulpatov, Izv. Vuzov Radiofiz. 10 (1967) 1414.

[5] N.S. Erokhin et al., Non-quilibrium and resonance processes in a plasma radiophysics, Moscow (1982) pp. $3-56$.

[6] V.L. Bratman, N.S. Ginzburg and M.I. Petelin, Opt. Commun. 30 (1979) 409

[7] M.V. Fjodorov, Usp. Fiz. Nauk 135 (1981) 213.

[8] R.L. Swent, R.N. Pantell and M.J. Alguard, Nucl. Instrum. Methods 170 (1980) 7.

[9] V.G. Baryshevsky and I.Ya. Dubovskaya, Phys. Stat. Sol. 82b (1977) 403. 